\title{
Positive Lyapunov Exponents for Schrödinger Operators with Quasi-Periodic Potentials
}

\section{Eugene Sorets and Thomas Spencer}

Institute for Advanced Study, Princeton, NJ 08540, USA

Received February 11, 1991; in revised form May 6, 1991

Abstract. We present a new, simple way to estimate the rate of exponential growth (Lyapunov exponent) of solutions of the finite-difference Schrödinger equation:

$$
((H-E) \psi)(n) \stackrel{\text { def }}{=}-[\psi(n+1)+\psi(n-1)]+[\lambda f(\alpha n+\theta)] \psi(n) .
$$

Here $f$ is a non-constant real-analytic function of period 1 and $\alpha$ is irrational. For $\lambda$ large we prove that the Lyapunov exponent is positive for every energy $E$ in the spectrum of $H$ and a.e. $\theta$. In particular, the absolutely continuous spectrum of $H$ is empty. In the continuum we study the quasi-periodic operator on $L^{2}(R)$

$$
H=-\frac{d^{2}}{d x^{2}}-K^{2}[\cos x+\cos (\alpha x+\theta)]
$$

for large $K$ and show that for wide intervals of low energies the Lyapunov exponent is positive. The main idea, which originated from M. Herman's subharmonic argument [11], is to deform the phase $\theta$ to the complex plane. This enables us to avoid small denominator problems by moving them off the axis, making estimates much easier to perform. We recover the information for real $\theta$ using an elementary extension of Jensen's formula (subharmonicity).

\section{Table of Contents}

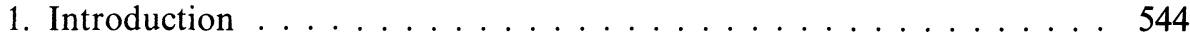

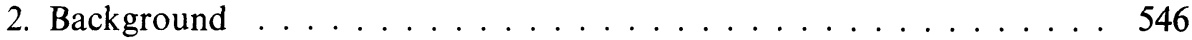

3. The Lattice Case . . . . . . . . . . . . . . . . . . . . . . 548

4. Differential Operator $H_{c} \ldots \ldots \ldots \ldots \ldots \ldots \ldots \ldots \ldots$

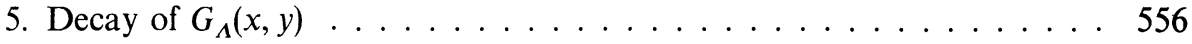

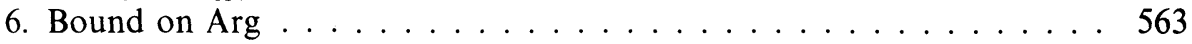

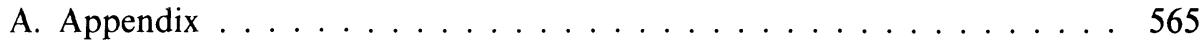

A.1 Proof of Proposition $3 \ldots \ldots \ldots \ldots \ldots \ldots$

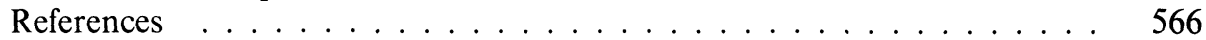




\section{Introduction}

In this paper we shall study the operator on $L^{2}(\mathbb{R})$

$$
H_{c}(\theta)=-D^{2}+V
$$

where $D^{2}=d^{2} / d x^{2}$ with the potential $V=-K^{2}[\cos x+\cos (\alpha x+\theta)]$, and its discrete analogue on $l^{2}(\mathbb{Z})$

$$
H(\theta)=-\Delta+V
$$

In the discrete case $\Delta$ denotes the finite-difference Laplacian $(\Delta g)(n) \stackrel{\text { def }}{=} g(n+1)+$ $g(n-1)$ and the potential is given by $V(n)=\lambda f(\alpha n+\theta)$, where $f$ is a real-analytic function of period 1 with $f(\mathbb{R})=[-1,1]$. The coupling constants $K$ and $\lambda$ are assumed to be real and the parameter $\theta$ is the phase. Notice that if $\alpha$ is rational, then $V$ in both cases is periodic. It is a standard result in Floquet theory that for all values of the coupling constants $K$ and $\lambda$ the spectra of the above operators consist of bands of purely absolutely continuous spectrum with generalized eigenfunctions of the form $(a+b x) e^{i c x} p(x)$, where $p(x)$ is periodic. In particular, if $E$ is in the spectrum of $H$ the Lyapunov exponent $\gamma(E)$, which measures the exponential rate of growth of $\psi$, vanishes. For more information see [17].

When $\alpha$ is irrational, the potential $V$ is quasi-periodic and the underlying dynamical system $\theta \mapsto \theta+\alpha$ is ergodic. As a consequence, both absolutely continuous and point spectrum are independent of $\theta$ for almost all $\theta$ [13]. In 1975 Dinaburg and Sinai [7] used K.A.M. analysis to prove that the behavior observed for rational $\alpha$ persists for most high energies. More precisely, they showed that if $\alpha$ is diophantine (poorly approximated by rationals) then for any $K, \sigma_{\mathrm{ac}}\left(H_{c}\right) \neq \varnothing$ and that there are eigenfunctions of the form $e^{i a x} q p(x)$ with $q p(x)$ quasi-periodic. However, they did not exclude the coexistence of point or singular continuous spectrum. S. Surace [22] later showed that for small $K$ there is no point spectrum. Corresponding results for $H$ were proved by Delyon [6]. Recent work concerning the absence of singular continuous spectrum can be found in [3,8 and 1].

For large $\lambda$, Sinai [19] and, independently, Fröhlich, Spencer, and Wittwer [10], proved that for diophantine $\alpha$ the spectrum of $H$ is pure point with exponentially localized eigenstates. Similar results hold in the continuum at low energies for $H_{c}$ [10]. The above authors worked with $H$ by analyzing its Green's function $G(E)=$ $(H-E)^{-1}$. When the eigenvalues of $H$ come close to $E$ the Green's function becomes singular. In order to control these "small divisors" they employed a multiscale perturbation scheme of K.A.M. type, which involved difficult induction arguments.

In this paper we first study $H$ and prove that for large $\lambda$ the Lyapunov exponent $\gamma(E, \theta)>0$ for every energy $E$ for a.e. $\theta$. This implies, in particular [16], that $H$ has no absolutely continuous spectrum. We prove a similar result for $H_{c}$ at low energies. More precisely, we show that there is a set $\mathscr{E}$ composed of intervals of width $K$ separated by $O\left(K^{-2}\right)$ such that for $E \in \mathscr{E}, \gamma(E) \sim K$. Moreover, $\mathscr{E} \cap \sigma(H) \neq \varnothing$. For precise statements see Theorems 2 and 4 . In fact, our methods actually can establish positivity of the Lyapunov exponent for all low energies, for $K$ large enough, but we do not present details here. 
We do not assume that $\alpha$ is diophantine - only that it is irrational. The price we pay for this freedom is that we can only prove the absence of absolutely contiruous spectrum; our methods do not distinguish between point and singular continuous spectra. In fact, for $\alpha$ Liouville $H$ has purely singular continuous spectrum when $f=\cos$ and $\lambda>2[9,20]$.

Although the results of our method are not as detailed as those in [10] or [19], and we require analyticity, its advantage lies in its simplicity and it allows some generalizations which would be difficult with the K.A.M.-type methods. For example, extension to the case of several frequencies is relatively straightforward with our method and is quite difficult even for two frequencies [4] using other techniques. In addition, the range of constants is improved significantly: in the case of $H$ with $V_{n}(\theta)=2 \lambda \cos (2 \pi(\theta+\alpha n))+\varepsilon f(\alpha n+\theta)$ we can prove the absence of absolutely continuous spectrum for $\lambda>1$ arbitrarily close to 1 provided $f$ is sufficiently regular and $\varepsilon$ is small. This is best one can expect [6] since when $\lambda<1$, and $\varepsilon=0$ a duality argument implies that all the spectrum is continuous. By contrast, the K.A.M.-based methods typically require $\lambda$ to be extremely large.

Recently, these methods were extended by I. Goldsheid and E.S. to potentials on strips. Those results will be published separately.

Our analysis of these quasi-periodic potentials was inspired by the work of Michael Herman on diffeomorphisms of the torus [11], where he considered the case $f=$ cos. We explain his result in Sect. 3 below.

In order to prove that the solutions of $\left(H_{c}(\theta)-E\right) \psi=0$ grow we prove that the Green's function $G(x, y, E, \theta)$ decays. To do that we first consider $H_{c}(\theta)$ for $\theta$ complex. The effect of moving $\theta$ into $\mathbb{C}$ is to move some of the spectrum of $H$ off the real axis (see Fig. 1) making $G$ a bounded operator, thereby eliminating the "small divisors" mentioned above. We can then apply a WKB type argument to obtain the decay of $G$ for $\theta$ complex. The information about $G(E, \theta)$ for $\theta \in \mathbb{R}$ is recovered using a subharmonic argument. See the next section and $[11,5]$.

Some time ago, P. Sarnak [18] studied families of non-self-adjoint operators closely related to $H(\theta)$ for complex $\theta$ and obtained pictures very similar to Fig. 1. These pictures suggest that localization starts in the middle of the spectrum and extends outward as $\lambda$ (or $K$ ) increases. This phenomenon was observed in numerical studies [12].

The rest of the paper is organized as follows. In the next section we introduce some background from ergodic theory and describe the subharmonic argument (an extension of Jensen's formula) that relates the decay rates of $G$ for real and complex $\theta$. In Sect. 3 we study $H$ which is technically much simpler - we do not even need to work with $G$, but estimate the rate of growth of the solution directly. Section 4 introduces some more background necessary for the continuum case, and in Sect. 5 we prove that for complex $\theta$ the Green's function decays using block-resolvent expansion and WKB analysis. Finally, in Sect. 6 we compute a bound on $\operatorname{Arg} G$, which arises in our version of Jensen's formula.

Fig. 1. The spectrum of $H_{c}(\theta)$ (left) and $H_{c}(\theta+i \delta)$ at low energies 


\section{Background}

To analyze the spectra and the Lyapunov exponent of $H$, we consider the finitedifference equation for the generalized eigenfunction $\psi$ corresponding to any $E$. Let $\psi$ be a solution of the equation $H \psi=E \psi$ and let

$$
\Psi_{n}=\left[\begin{array}{c}
\psi_{n+1} \\
\psi_{n}
\end{array}\right]
$$

Then $\Psi_{n}=M_{n} \Psi_{n-1}=P_{n} \Psi_{0}$, where

$$
M_{n}(\theta)=\left[\begin{array}{cc}
\lambda V_{n}(\theta)-E & -1 \\
1 & 0
\end{array}\right] \text { and } P_{n}=M_{n} \cdots M_{1} .
$$

Note that Det $M_{n}=1$. We choose $V_{n}$ to be:

$$
V_{n}(\theta)=V_{0}\left[R_{\alpha}^{n}(\theta)\right]
$$

where $V_{0}\left[R_{\alpha}^{n}(\theta)\right]$ is a real-analytic function of period 1 , and $R_{\alpha}: S^{1} \rightarrow S^{1}$ is the rotation by $\alpha$ :

$$
R_{\alpha}(\theta)=\theta+\alpha .
$$

Then $P_{n}(\theta)$ is a product of the values of a matrix-valued function $\theta \mapsto M_{0}(\theta)$ evaluated along the orbit of $\theta$ under the action of $R_{\alpha}$. Note that since $\alpha$ is irrational $R_{\alpha}$ is ergodic. One consequence of ergodicity is that all the spectrum of $H$ is essential with no isolated eigenvalues and $\sigma_{\mathrm{ac}}(H), \sigma_{p}(H)$, and $\sigma_{s}(H)$ are independent of almost every $\theta$.

The Lyapunov exponent $\gamma(E, \theta)$ is defined by:

$$
\gamma(E, \theta)=\lim _{N \rightarrow \infty} \frac{1}{N} \log \left\|P_{N}(E, \theta)\right\| .
$$

It measures the average rate of growth of the solution $\psi$. Existence of $\gamma$ is guaranteed by the Subadditive Ergodic Theorem:

Theorem 1. (Subadditive Ergodic Theorem) [14]. Let $(X, \mathscr{B}, m)$ be a probability space and let $T: X \rightarrow X$ be measure-preserving. Let $\left\{f_{n}\right\}_{1}^{\infty}$ be a sequence of measurable functions $f_{n}: X \rightarrow \mathbb{R} \cup\{-\infty\}$ satisfying the conditions:

(a) $f_{1}^{+} \in L^{1}(m)$

(b) for each $k, n \geqq 1 f_{n+k} \leqq f_{n}+f_{k} \circ T^{n}$ a.e.

Then there exists a measurable function $f: X \rightarrow \mathbb{R} \cup\{-\infty\}$ such that

and

$$
f^{+} \in L^{1}(m), \quad f \circ T=f \text { a.e., } \quad \lim _{n \rightarrow \infty} \frac{1}{n} f_{n}=f \text { a.e., }
$$

$$
\lim _{n \rightarrow \infty} \frac{1}{n} \int f_{n} d m=\inf _{n} \frac{1}{n} \int f_{n} d m=\int f d m .
$$

We take $S^{1}$ with normalized Lebesque measure and $R_{\alpha}$ as our dynamical system and $f_{n}=\log \left\|P_{n}\right\|$. It is clear that the hypotheses of the theorem are satisfied. 
Moreover, since $R_{\alpha}$ is ergodic $\gamma(E, \theta)$ is constant for a.e. $\theta$ and

$$
\gamma(E)=\gamma(E, \theta)=\int_{0}^{1} \gamma(E, \theta) d \theta=\inf _{N} \frac{1}{N} \int_{0}^{1} \log \left\|P_{N}(E, \theta)\right\| d \theta .
$$

In the sequel we shall prove that for every $E$ the Lyapunov exponent is positive for a.e. $\theta$.

We shall now explain Herman's result. Let $z=e^{2 \pi i \theta}$ and write

$$
V(n, \theta)=2 \lambda \cos 2 \pi(\alpha n+\theta)=\lambda e^{2 \pi i \alpha n} z+\lambda e^{-2 \pi i \alpha n} \frac{1}{z} \stackrel{\text { def }}{=} V(n, z) .
$$

It follows from (2.2) that

$$
z^{N} P_{N}=\prod_{n=1}^{N}\left[\begin{array}{cc}
\lambda e^{-2 \pi i \alpha n}+\lambda e^{2 \pi i \alpha n} z^{2}-E z & -z \\
z & 0
\end{array}\right]
$$

is an entire function of $z$, and, hence, $\log \left\|z^{N} P_{N}\right\|$ is subharmonic. Since $|z|=1$,

$$
\begin{aligned}
\gamma(E) & =\inf _{N} \frac{1}{N} \int \log \left\|P_{N}(E, \theta)\right\| d \theta \\
& =\inf _{N} \frac{1}{N} \int \log \left\|z^{N} P_{N}(E, \theta)\right\| d \theta \\
& \geqq\left.\frac{\log \left\|z^{N} P_{N}(z)\right\|}{N}\right|_{z=0}=\frac{1}{N} \log \lambda^{N}=\log \lambda .
\end{aligned}
$$

Hence, $\gamma>0$ whenever $\lambda>1$.

The limitation of Herman's approach is that it breaks down under perturbations. If $\lambda \cos 2 \pi(\alpha n+\theta)$ is replaced by $\lambda \cos 2 \pi(\alpha n+\theta)+\varepsilon \cos 4 \pi(\alpha n+\theta)$, the bound becomes $\gamma>\log \varepsilon$. We get around this problem with the help of an extension of Jensen's formula. We include a proof for completeness.

Definition 1. By $\mathscr{A}\left(r_{1}, r_{2}\right)$ we shall denote the annulus $\left\{z \in \mathbb{C}\left|r_{1}<\right| z \mid<r_{2}\right\}$.

Lemma 1. Let $g$ be meromorphic on $\mathscr{A}=\mathscr{A}(r, 1)$ and continuous on $\overline{\mathscr{A}}$, and let $\left\{r_{i}\right\}$ and $\left\{p_{j}\right\}$ be the roots and poles of $g$ such that $r<\left|r_{i}\right|,\left|p_{j}\right| \leqq 1$.

Then for $z=r e^{2 \pi i \theta}$ :

$$
\int_{0}^{1} \log \left|g\left(e^{2 \pi i \theta}\right)\right| d \theta=\sum_{p_{J} \in \mathscr{A}} \log \left|p_{j}\right|+\sum_{r_{t} \in \mathscr{A}} \log \frac{1}{\left|r_{i}\right|}+\int_{0}^{1} \log \left|g\left(r e^{2 \pi i \theta}\right)\right| d \theta+(\underset{|z|=r}{\operatorname{Arg} g}) \log \frac{1}{r}
$$

where

$$
\underset{|z|=r}{\operatorname{Arg}} g=\frac{1}{2 \pi i} \int_{|z|=r} \frac{g^{\prime}}{g}(z) d z .
$$

Proof. Let $h$ be the non-vanishing analytic function defined by $g(z)=$ 


$$
\begin{aligned}
& \Pi\left(z-p_{j}\right)^{-1} \Pi\left(z-r_{i}\right) h(z) \text {. Then: } \\
& \begin{aligned}
\int_{0}^{1} \log \left|g\left(e^{2 \pi i \theta}\right)\right| d \theta & =\sum_{r_{i}}^{1} \int_{0}^{1} \log \left|e^{2 \pi i \theta}-r_{i}\right| d \theta-\sum_{p_{j}} \int_{0}^{1} \log \left|e^{2 \pi i \theta}-p_{j}\right| d \theta+\int_{0}^{1} \log \left|h\left(e^{2 \pi i \theta}\right)\right| d \theta \\
& =\int_{0}^{1} \log \left|h\left(e^{2 \pi i \theta}\right)\right| d \theta
\end{aligned}
\end{aligned}
$$

In the last equality we used the fact that

$$
\int_{0}^{1} \log \left|e^{2 \pi i \theta}-a\right| d \theta=\int_{0}^{1} \log \left|1-a e^{2 \pi i \theta}\right| d \theta=\Re \frac{1}{2 \pi i} \int_{|z|=1} \log (1-a z) \frac{d z}{z}=0,
$$

where $a=p_{j}$ or $r_{i}$ and $|a|<1$. Similarly,

$$
\int_{0}^{1} \log \left|g\left(r e^{2 \pi i \theta}\right)\right| d \theta=\sum_{p_{j} \in \mathscr{A}} \log \left|p_{j}\right|+\sum_{r_{1} \in \mathscr{A}} \log \frac{1}{\left|r_{i}\right|}+\int_{0}^{1} \log \left|h\left(r e^{2 \pi i \theta}\right)\right| d \theta .
$$

Since $\log h$ is analytic in the slit annulus, $\underset{|z|=t}{\operatorname{Arg} h}$ is constant for $t \in[r, 1]$, and

$$
\begin{aligned}
& \int_{0}^{1} \log \left|h\left(e^{2 \pi i \theta}\right)\right| d \theta-\int_{0}^{1} \log \left|h\left(r e^{2 \pi i \theta}\right)\right| d \theta \\
& =\mathfrak{R} \int_{r}^{1} \frac{d t}{t} \underset{|z|=t}{\operatorname{Arg}} h=(\underset{|z|=r}{\operatorname{Arg} h}) \log \frac{1}{r}=(\underset{|z|=r}{\operatorname{Arg} g}) \log \frac{1}{r} .
\end{aligned}
$$

In the last equality we used $\operatorname{Arg} g=\operatorname{Arg} \Pi\left(z-p_{j}\right)^{-1} \Pi\left(z-r_{i}\right)+\operatorname{Arg} h$ and $\left|p_{j}\right|$, $\left|r_{i}\right|>r$ :

We cannot apply Jensen's formula to $\left\|P_{N}\right\|$ directly, but we note that

$$
\left\|P_{N}\right\| \geqq\left|\left\langle\left(\begin{array}{l}
1 \\
0
\end{array}\right), P_{N}(z)\left(\begin{array}{l}
1 \\
0
\end{array}\right)\right\rangle\right| \stackrel{\text { def }}{=}\left|g_{N}(z)\right|
$$

and that $g_{N}(z)$ is analytic in $z$ in an annulus determined by regularity of $f$. It is to $g_{N}$ on that annulus that we apply the Jensen's formula. Since there are no poles and contribution from the roots is nonnegative we have an inequality:

$$
\int_{|z|=1} \log \left|g_{N}\right| d \theta \geqq \int_{|z|=r} \log \left|g_{N}\right| d \theta+\left(\underset{|z|=r}{\operatorname{Arg}} g_{N}\right) \log \frac{1}{r} .
$$

The estimates for the right-hand side are done in the next section.

The treatment of the operator $H_{c}$ is similar, but more complicated. See Sects. 4-6.

\section{The Lattice Case}

Our first main theorem is:

Theorem 2. Let $H=-\Delta+V$ with $V_{n}(\theta)=f(\alpha n+\theta)$ for $f$ real-analytic with period 1 such that $f(\mathbb{R})=[-1,1]$. 
Then, there exists $\lambda_{0}$ such that for every $\lambda>\lambda_{0}$,

$$
\gamma_{E}(\theta)>0
$$

for every $E$ and a.e. $\theta$.

We shall first establish this theorem in the special case $V_{0}(\theta)=2 \lambda \cos 2 \pi \theta+\varepsilon f(\theta)$ with $f$ analytic of period 1 in $\mathscr{A}(r, 1)$ as in Lemma 1 .

Theorem 3. Let $V_{0}$ be as above and $H$ be as in (1.2), with $\alpha$ irrational. Given $E$ and $\beta>0$ we assume for $|z|=r$,

$$
\left|\lambda+\lambda z^{2}-E z\right|>1+2 \beta+|z|
$$

and let $\varepsilon_{0}(\lambda)=\frac{\beta}{\sup _{\mathscr{A}}|f|}$.

Then for $|\varepsilon|<\varepsilon_{0}(\lambda)$ the Lyapunov exponent

$$
\gamma=\gamma(E)>\log (1+\beta) \text {. }
$$

Corollary 1. If $\lambda>1$ and $\delta>0$, then there exist positive $r_{0}$ and $\varepsilon_{0}$ such that for $0 \leqq r<r_{0}$ and $|\varepsilon|<\varepsilon_{0}$,

$$
\gamma(E)>\log (\lambda-\delta)
$$

for all $|E|<2 \lambda+2$. In particular, (3.4) holds for all $E \in \sigma(H)$.

Remark 1. It follows from the results of Pastur [16] that $H$ has no absolutely continuous spectrum on any interval of energies where $\gamma(E)>0$.

Again, let $z=e^{2 \pi i \theta}$ so that $r \stackrel{\text { def }}{=}|z|=e^{2 \pi i \Im \theta}$. Let $P_{N}$ be as in (2.2) and

$$
F_{N}(z) \stackrel{\text { def }}{=}\left(\prod_{k=1}^{N} z_{k}\right) P_{N}(z)
$$

where $z_{k}=e^{2 \pi i \alpha k} z$ so that

$$
F_{N}(z)=\prod_{n=1}^{N}\left[\begin{array}{cc}
\lambda+\lambda z_{k}^{2}-E z_{k}+\varepsilon z_{k} f\left(z_{k}\right) & -z_{k} \\
z_{k} & 0
\end{array}\right]
$$

is analytic on $\mathscr{A}$. Also let

$$
h_{N}(z)=\left(\prod_{k=1}^{N} z_{k}\right) g_{N}(z)
$$

for $g_{N}(z)$ as in (2.21). From (2.21), (2.16), and (2.22), we get:

$$
\begin{aligned}
\int \log \left\|P_{N}\left(e^{2 \pi i \theta}\right)\right\| d \theta & \geqq \int \log \left|g_{N}\left(e^{2 \pi i \theta}\right)\right| d \theta \\
& =\int \log \left|h_{N}\left(e^{2 \pi i \theta}\right)\right| d \theta \\
& \geqq \int \log \left|h_{N}\left(e^{2 \pi i \theta}\right)\right| d \theta+\left(\underset{|z|=r}{\operatorname{Arg} h_{N}}\right) \log \frac{1}{r} .
\end{aligned}
$$

Therefore, in order to prove that $\gamma(E)>0$, we need only show that the right-hand side grows linearly with $N$. The estimate is the content of Propositions 1 and 2 below. 
Proposition 1. Suppose that $f$ is analytic on $\mathscr{A}\left(r_{0}, 1\right)$ for some $r_{0} \in[0,1)$ and for some $\beta>0$ and $r \in\left[r_{0}, 1\right)$,

$$
\inf _{|z|=r}\left|\lambda+\lambda z^{2}-E z+\varepsilon z f(z)\right|>1+\beta+r
$$

Then for all $N \in \mathbb{Z}$

$$
\left|h_{N}(z)\right|>(1+\beta)^{N}
$$

for $|z|=r$.

Proof. For shorthand, let

$$
A_{k}=\lambda+\lambda z_{k}^{2}-E z_{k}+\varepsilon z_{k} f\left(z_{k}\right) .
$$

Then $F_{N}$ in (3.6) applied to a vector $\left(1, \eta_{1}\right)^{t}$ can be written as:

$$
\left[\begin{array}{cc}
A_{N} & -z_{N} \\
z_{N} & 0
\end{array}\right] \cdots\left[\begin{array}{cc}
A_{1} & -z_{1} \\
z_{1} & 0
\end{array}\right]\left[\begin{array}{c}
1 \\
\eta_{1}
\end{array}\right] .
$$

It is easy to prove by induction that (3.12) equals

$$
\prod_{1}^{N}\left(A_{k}-z_{k} \eta_{k}\right)\left[\begin{array}{c}
1 \\
\eta_{N}
\end{array}\right]
$$

where

$$
\eta_{k+1}=\frac{1}{A_{k} / z_{k}-\eta_{k}} .
$$

If $\left|\eta_{1}\right|<1$, then by Eqs. (3.9) and (3.14) $\left|\eta_{k}\right|<1$ for all $k>1$ and

$$
\left|A_{k}-z_{k} \eta_{k}\right| \geqq\left|A_{k}\right|-\left|z_{k}\right| \geqq 1+\beta+r-r \geqq 1+\beta .
$$

It follows that

$$
\left|h_{N}(z)\right| \geqq \prod_{1}^{N}\left|A_{k}-z_{k} \eta_{k}\right|\left|\left\langle\left(\begin{array}{c}
1 \\
0
\end{array}\right),\left(\begin{array}{c}
1 \\
\eta_{N}
\end{array}\right)\right\rangle\right| \geqq(1+\beta)^{N} .
$$

Proposition 2. Let $h_{N}$ be given by (3.7) and (2.21) and $\mathscr{A}, \lambda, z, r_{0}$, and $r$ be as in Proposition 1. Then

$$
\underset{|z|=r}{\operatorname{Arg}} h_{N}=0
$$

Proof. When $\varepsilon=0 h_{N}(z)$ is entire and doesn't vanish on $\{|z|<r\}$ by Proposition 1 . It follows that (with $\varepsilon=0$ )

$$
\underset{|z|=r}{\operatorname{Arg}} h_{N}=0
$$

Since Proposition 1 continues to hold with $|\varepsilon|<\varepsilon_{0}$, the roots of $h_{N}$ do not cross $|z|=r$ and thus Arg does not change.

We are now ready to prove Theorem 3.

Proof of Theorem 3. Note that the hypotheses of Theorem 3 imply that (3.9) holds. The subadditive ergodic theorem, (2.21), (2.16), and Propositions 1 and 2 imply 
that

$$
\begin{aligned}
\gamma(E) & \geqq \inf _{N} \int_{0}^{1} \frac{1}{N} \log \left|g_{N}\left(e^{2 \pi i \theta}\right)\right| d \theta \\
& =\inf _{N} \int_{0}^{1} \frac{1}{N} \log \left|h_{N}\left(e^{2 \pi i \theta}\right)\right| d \theta \\
& \geqq \inf _{N} \int_{0}^{1} \frac{1}{N} \log \left|h_{N}\left(r e^{2 \pi i \theta}\right)\right| d \theta \\
& \geqq \log (1+\beta)>0 .
\end{aligned}
$$

The argument is essentially the same when the potential is

$$
V_{n}(\theta)=f(\alpha n+\theta)
$$

with $f$ real-analytic with period 1 as in Theorem 2 .

Proof of Theorem 2. By the analyticity of $f$ we can choose $r$ arbitrarily near 1 such that on $\{|z|=r\}$

$$
\left|f-\frac{E}{\lambda}\right| \geqq m_{0}>0
$$

where $E$ and $\lambda$ are from Theorem 2 . The positive number $m_{0}$ can be seen to depend on $f$ only. Then $|\lambda f-E|>m_{0} \lambda$ and with $h$ defined as in (3.7) we have

$$
\left|h_{N}(z)\right| \geqq \prod_{1}^{N}\left(\left|A_{k}\right|-r\right)>r^{N}\left(m_{0} \lambda-1\right)^{N}
$$

for $\lambda>\lambda_{0} \stackrel{\text { def }}{=} 3 / m_{0}$ as in Proposition 1 .

Also, for $\lambda>\lambda_{0}$

$$
\underset{|z|=r}{\operatorname{Arg}} h_{N}(z)=\operatorname{Arg} \prod_{1}^{N}\left(A_{k}-z_{k} \eta_{k}\right)=\sum_{1}^{N} \operatorname{Arg} A_{k}=N+N \operatorname{Arg}(\lambda f-E) .
$$

It follows that

$$
\begin{gathered}
\gamma(E) \geqq \log r\left(m_{0} \lambda-1\right)+\left(\log \frac{1}{r}\right)(1+\operatorname{Arg}(\lambda f-E)) \\
=\log \left(m_{0} \lambda-1\right)+\left(\log \frac{1}{r}\right) \operatorname{Arg}(\lambda f-E)>0
\end{gathered}
$$

for $\lambda$ sufficiently large since $\operatorname{Arg}(\lambda f-E)$ is independent of $\lambda>\lambda_{0}$.

\section{Differential Operator $H_{c}$}

In the next three sections we shall prove an equivalent of Theorem 3 for the operator $H_{c}$, but we are going to exclude some narrow energy intervals from consideration. The set $\mathscr{E}$ of admissible energies will be composed of intervals of width $K$ separated by $O\left(K^{-2}\right)$. The precise definition is given in the next section. See Definition 3. 
Our theorem in the continuum is:

Theorem 4. Let $H=H(\theta)$ be as in (1.1) and $\mathscr{E}$ as in Definition 3. Then for $E \in \mathscr{E}$ and sufficiently large $K$

$$
\gamma(E) \geqq K+o(1)
$$

Since $\gamma(E)$ is always positive for $E \notin \sigma\left(H_{c}\right)$, we need to know that our result is not vacuous.

Proposition 3. Let an interval $I \subset\left[\inf \sigma\left(H_{c}\right), \inf \sigma\left(H_{c}\right)+200 K\right]$ be longer than $e^{-K^{1 / 6}}$. Then

Proof. See appendix.

$$
\sigma\left(H_{c}\right) \cap I \neq \varnothing
$$

We are going to prove that $\gamma(E)>0$ for intervals of energies of length const. $K$ which by Proposition 3 must contain energies from the spectrum of $H_{c}$. The basic idea is still the same - we will use subharmonicity - but the analysis will be different. The role of $P_{N}$ is now played by the fundamental matrix $\Phi=\Phi_{\theta}$ given by

$$
\Phi_{\theta}^{\prime}(x)=\left[\begin{array}{cc}
0 & V_{\theta}(x) \\
1 & 0
\end{array}\right] \Phi_{\theta}(x), \quad \Phi\left(x_{0}\right)=I .
$$

The underlying dynamical system is the flow $R_{\alpha}^{x}$ on the torus $\mathbb{T}^{2}$ :

$$
R_{\alpha}^{x}(u, v)=(u+x, v+\alpha x) .
$$

In terms of $R_{\alpha}^{x}$ the potential

$$
V_{\theta}(x)=-K^{2}[\cos x+\cos (\alpha x+\theta)]=-K^{2}\left[\cos \pi_{1}\left(R_{\alpha}^{x}(0,0)\right)+\cos \pi_{2}\left(R_{\alpha}^{x}(0, \theta)\right)\right],
$$

where $\pi_{i}$ denotes the projection onto the $i^{\text {th }}$ coordinate of $\mathbb{T}^{2}$. Lyapunov exponent $\gamma=\gamma(E, \theta)$ is defined as before:

$$
\gamma(E, \theta)=\lim _{T \rightarrow \infty} \frac{1}{T} \log \left\|\Phi_{\theta}(T)\right\| .
$$

Since $\alpha$ is irrational, $R_{\alpha}$ is ergodic. It follows that for a.e. $\theta$,

$$
\begin{aligned}
\gamma(E, \theta)=\gamma(E) & =\frac{1}{2 \pi} \int_{0}^{2 \pi} \gamma(E, \theta) d \theta \\
& =\lim _{T \rightarrow \infty} \int_{0} \frac{1}{T} \log \left\|\Phi_{\theta}(T)\right\| d \theta \\
& =\inf _{T} \int_{T} \frac{1}{T} \log \left\|\Phi_{\theta}(T)\right\| d \theta .
\end{aligned}
$$

As in the lattice case we shall obtain lower bounds on

$$
\int \frac{1}{T} \log \left\|\Phi_{\theta}(T)\right\| d \theta
$$

We do that by studying the Green's function of the deformed operator $H_{c}$. 
Connection between decay of the deformed and the undeformed $G$ is provided, as before, by Jensen's formula.

The structure of the argument is the following. Lemmas 2 and 3 below say that for every $\varepsilon, \gamma_{\varepsilon} \stackrel{\text { def }}{=} \gamma(E+i \varepsilon)$ is at least the decay rate of $G(E+i \varepsilon)$. We prove in Sects. 5 and 6 that for every $E \in \mathscr{E}$ (to be specified later) for almost every $\theta, \gamma_{\varepsilon} \geqq K$ for all small $\varepsilon \neq 0$, and, since $\gamma_{\varepsilon}$ is an infimum of continuous functions of $\varepsilon$

$$
\gamma=\gamma_{0} \geqq K \text {. }
$$

Lemma 2. Suppose that for all sufficiently large $x$

$$
\left|G\left(x_{0}, x, E+i \varepsilon\right)\right| \leqq A e^{-b\left|x-x_{0}\right|},
$$

where $G$ is the Green's function of $H_{c}$, and $A$ and $b$ are positive constants independent of $x$. Then there is a constant $B \in \mathbb{R}^{+}$such that

$$
\left\|\Phi^{\varepsilon}(x)\right\|>B e^{b\left|x-x_{0}\right|} .
$$

Proof. Let $\phi(x) \stackrel{\text { def }}{=} G\left(x_{0}, x, E+i \varepsilon\right)$ for $x>x_{0}$ and let $\psi$ be another solution of $u^{\prime \prime}=(V-E) u$ such that

$$
\left(\phi \psi^{\prime}-\phi^{\prime} \psi\right)\left(x_{0}\right)=1
$$

Since

$$
\left|\phi^{\prime \prime}(\xi)\right|=|(V(\xi)-E) \phi(\xi)| \leqq 2 K^{2}|\phi(\xi)|
$$

for all $\xi$, expanding $\phi(x+1)$ to second order in Taylor series about $x$, we get:

$$
\left|\phi^{\prime}(x)\right|<\left(2 A+K^{2}\right) e^{-b\left|x-x_{0}\right|} .
$$

The Wronskian in (4.11) is independent of $x$ (so long as $x>x_{0}$ ), and so

$$
1=\left|\left(\phi \psi^{\prime}-\phi^{\prime} \psi\right)(x)\right| \leqq|\phi|\left|\psi^{\prime}\right|+\left|\phi^{\prime}\right||\psi| \leqq\left(2 A+K^{2}\right) e^{-b\left|x-x_{0}\right|}\left(|\psi|+\left|\psi^{\prime}\right|\right) .
$$

It follows that

$$
|\psi|+\left|\psi^{\prime}\right| \geqq \frac{e^{b\left|x-x_{0}\right|}}{2 A+K^{2}} .
$$

The case when $x<x_{0}$ is similar.

For the remainder of the paper we are going to drop the subscript $c$ in $H_{c}$, and we shall frequently emphasize dependence on $\theta$ or $z=e^{2 \pi i \theta}$ by writing $H(\theta)$ or $H(z)$. Let the real and imaginary parts of $H$ be given by

$$
\mathfrak{R} H \stackrel{\text { def }}{=}-D^{2}+\mathfrak{R} V \text { and } \mathfrak{I} H \stackrel{\text { def }}{=} \mathfrak{I} V .
$$

For any interval $I$, by $H_{I}$ we shall denote the restriction of $H$ to $L^{2}(I)$ with Dirichlet boundary conditions and $G_{I}(E) \stackrel{\text { def }}{=}\left(H_{I}-E\right)^{-1}$. Throughout the discussion we are going to make frequent use of the "resolvent identity," which is the one-dimensional analogue of the Poisson Integral formula. Let $R \subset \Lambda$ be any two intervals and let $G_{\Lambda, R} \stackrel{\text { def }}{=} G_{\Lambda \backslash R} \oplus G_{R}$. Then

$$
G_{\Lambda}(x, y)=G_{\Lambda, R}(x, y)+\sum_{r \in \partial \Lambda \backslash \partial R} G_{\Lambda, R}^{\bullet}(x, r) G_{\Lambda}(r, y),
$$


where $G^{\bullet}$ is the normal derivative of $G$ with respect to $r$. Note that if $x, y \in R$ then $G_{\Lambda, R}(x, y)=G_{R}(x, y)$ and if $\partial R$ separates $x$ and $y$ then $G_{\Lambda, R}(x, y)=0$.

Lemma 3. For every $\varepsilon \neq 0$,

$$
\lim _{x \rightarrow+\infty} \frac{\log |G(0, x, E+i \varepsilon, \theta)|}{x}
$$

exists for almost every $\theta$.

Proof. Let $\Lambda_{n}=(-\infty, 2 \pi(n+1)]$. Then by the resolvent identity

$$
\begin{aligned}
G(0, x)=G_{\Lambda_{1}}^{\bullet}(0,2 \pi) G(2 \pi, x) & =\prod_{n=1}^{[x]} G_{\Lambda_{n}}^{\bullet}(2 \pi(n-1), 2 \pi n, \theta) G\left(2 \pi\left[\frac{x}{2 \pi}\right], x\right) \\
& =\prod_{n=1}^{[x]} G_{\Lambda_{1}}^{\bullet}(0,2 \pi, \theta+2 \pi \alpha n) G\left(2 \pi\left[\frac{x}{2 \pi}\right], x\right) .
\end{aligned}
$$

It follows that

$$
\lim _{x \rightarrow+\infty} \frac{1}{x} \log |G(0, x)|=\lim _{x \rightarrow+\infty} \frac{1}{x} \sum_{n=1}^{[x]} \log \left|G_{\Lambda_{1}}^{\bullet}(0,2 \pi, \theta+2 \pi \alpha n)\right|
$$

exists by Birkoff's ergodic theorem.

To estimate the decay of $G(E+i \varepsilon)$ we first note that it is sufficient to prove that $G_{\Lambda}(x, y, E+i \varepsilon)$ decays for $x, y \in \Lambda$, where $\Lambda$ is an arbitrarily wide finite interval. We then estimate $G_{\Lambda}$ with the help of Jensen's formula.

The next two lemmas show that Jensen's formula is applicable.

Lemma 4. Let $G_{\Lambda}(x, y ; z)$ be the Green's function with Dirichlet boundary conditions in an interval $\Lambda$ for the operator $H_{\Lambda}(z)=-D^{2}+V(z)$, where $V$ is the potential from (1.1) written in terms of $z$ :

$$
V(x, z)=-K^{2}\left[\cos x+\frac{1}{2}\left(e^{i \alpha x} z+e^{-i \alpha x} \frac{1}{z}\right)\right] .
$$

Then $z \mapsto G_{\Lambda}(x, y ; z)$ is meromorphic.

Proof. We first prove that the operator $G_{\Lambda} \stackrel{\text { def }}{=}\left(H_{\Lambda}-E\right)^{-1}$ is meromorphic. Let $E \in \mathbb{C}$ be given. Since $\Lambda$ is a finite interval the spectrum of $H_{\Lambda}$ is discrete. Therefore, $H_{\Lambda}$ can be written as $H_{\Lambda}=F+R$, where

$$
F(z) \stackrel{\text { def }}{=} \frac{-1}{2 \pi i} \int_{C_{z}} \frac{H_{\Lambda} d \xi}{H_{\Lambda}(z)-\xi},
$$

the contour $C_{z}$ enclosing all eigenvalues of $H_{A}$ with absolute value less than $1+2|E| . F$, therefore, is a finite-dimensional operator, analytic in $z$. Since the spectrum $\sigma(R)$ of $R$ is at least distance $1+|E|$ from $E,(R-E)^{-1}$ exists and is analytic in $z$. Therefore, we can rewrite $H_{A}$ as

$$
\frac{1}{H_{\Lambda}-E}=\frac{1}{R-E+F}=\frac{1}{R-E} \cdot \frac{1}{1+(R-E)^{-1} F}
$$


and see that $\left(H_{A}-E\right)^{-1}$ is meromorphic in $z$. Indeed, $(R-E)^{-1}$ is analytic in $z$ and the only singularities the second factor can produce are the poles at the (finitely many) values of $z$ for which $-1 \in \sigma\left((R-E)^{-1} F\right)$.

Lemma 5. $G_{\Lambda}(x, y ; z) \neq 0$ for all $x, y \in \operatorname{Int} \Lambda$.

Proof. Suppose $G_{\Lambda}(x, y ; z)=0$ for some $x<y \in \mathbb{R}$ and $z \in \mathbb{C}$. Let $p \in \Lambda$ be less than $x$. Then by the resolvent identity we have:

$$
G_{\Lambda}(p, y)=G_{\Lambda_{x}}^{\bullet}(p, x) G_{\Lambda}(x, y)=0,
$$

where $\Lambda_{x}=\Lambda \cap(-\infty, x]$. That is, if $G_{\Lambda}(x, y)=0$, then $G_{\Lambda}(p, y)=0$ for all $p$ as above. But this is impossible since $G_{\Lambda}\left(p,{ }^{\circ}\right)$ is a non-trivial solution of the O.D.E. $u^{\prime \prime}=(V-E) u$ on $(p, \infty)$.

We now sketch the proof of Theorem 4.

Sketch of Proof. We shall prove that $G_{\Lambda}$ decays on sufficiently wide intervals $\Lambda$. Lemmas 2 and 3 will then imply that $\|\Phi\|$ grows exponentially; in other words, $\gamma(E)$ is positive.

Applying Jensen's formula to $G_{\boldsymbol{A}}$ we get:

$$
\begin{aligned}
\frac{1}{2 \pi} \int_{0}^{2 \pi} d \theta \log \left|G_{\Lambda}\left(x, y, E ; e^{i \theta}\right)\right|= & \frac{1}{2 \pi} \int_{0}^{2 \pi} d \theta \log \left|G_{\Lambda}\left(x, y, E ; r e^{i \theta}\right)\right| \\
& +\sum_{r<\left|r_{j}\right| \leqq 1} \log \left|\frac{1}{r_{j}}\right|+\sum_{r<\left|p_{j}\right| \leqq 1} \log \left|p_{j}\right| \\
& +\left(\underset{|z|=r}{\operatorname{Arg} G_{\Lambda}}\right) \log \frac{1}{r},
\end{aligned}
$$

where $r_{j}$ are the roots and $p_{j}$ are the poles of $G(x, y, E ; \cdot)$. Lemma 5 says that the first sum vanishes. The sum over the poles is non-positive, so we are left with the inequality:

$$
\frac{1}{2 \pi} \int_{0}^{2 \pi} d \theta \log \left|G_{\Lambda}\left(x, y, E ; e^{i \theta}\right)\right| \leqq \frac{1}{2 \pi} \int_{0}^{2 \pi} d \theta \log \left|G_{\Lambda}\left(x, y, E ; r e^{i \theta}\right)\right|+\left(\underset{|z|=r}{\operatorname{Arg}} G_{\Lambda}\right) \log \frac{1}{r} .
$$

The right-hand side of (4.25) is estimated with the help of the following two propositions, which are proved in Sects. 5 and 6, respectively. We shall restrict the values of $E$ to the set $\mathscr{E}$ defined in Sect. 5.

Proposition 4. For $\Lambda, E, x$, and $y$ as in Theorem 4 , and $1-K^{-5}=r$,

$$
\left|G_{\Lambda}\left(x, y, E ; r e^{i \theta}\right)\right| \leqq \text { const. } e^{-K|x-y|} \quad \forall \theta .
$$

Recall that $\delta=\log (1 / r)$.

Proposition 5. There is a constant independent of $x, y$, and $\Lambda$ such that

$$
\left|\left(\underset{|z|=r}{\operatorname{Arg}} G_{\Lambda}\right) \log \frac{1}{r}\right| \leqq \text { const. } \delta K^{2}|x-y||\log \delta| \text {. }
$$


The proof of Proposition 4 basically follows from WKB type estimates and since $\delta>0$ we can obtain apriori bounds on $G$ (Proposition 7) and this makes multiscale analysis unnecessary.

\section{Decay of $G_{A}(x, y)$}

In this section we prove that the Green's function with phase $\theta+i \delta$ for $\delta$ small decays exponentially fast. Our plan is to divide the $x$-axis into 2 kinds of regions: the wells and the decay regions. The wells $W$ are the neighborhoods of the local minima of $\Re V$ which are low enough to reach $\mathscr{E}$ - the set of energies that we consider. (See Definition 3 below.) In the wells the deformation will provide us with a priori bounds on $G_{W}$. (Proposition 7). Outside the wells - in the decay regions $-\mathfrak{R} V>\sup \mathscr{E}$ and we can use the WKB expansion to prove that $G$ decays there (Lemma 10). The information from all these regions is then patched together in Proposition 8 using block-resolvent expansion which expresses $G_{A}$ in terms of products of $G_{I}$, where $I$ is a well or a decay region.

In terms of $\theta$ and $\delta$ the potential $V$ is:

$$
\begin{aligned}
V(x, \theta+i \delta) & =-K^{2}[\cos x+\cos (\alpha x+\theta+i \delta)] \\
& =-K^{2}[\cos x+\cosh \delta \cos (\alpha x+\theta)]-i K^{2} \sinh \delta \sin (\alpha x+\theta) .
\end{aligned}
$$

A quick comparison with (4.18) yields the relation $\delta=-\log r$. $\delta$ will be chosen small, with an additional provison that $|\delta-\varepsilon|>\delta / 2$.

Definition 2. $A$ well $W$ is an interval of width $K^{-2 / 5}$ centered at $2 \pi k$ for $k \in \mathbb{Z} . H_{W}$ shall denote the operator $H$ with Dirichlet boundary conditions at $\partial W$.

We shall make use of the following fact.

Fact. Let $E_{n}$ be the $n^{\text {th }}$ eigenvalue of $H_{W}$ for the well centered at 0 . Then for $|\theta|<K^{-3 / 7}, n \leqq 100$ and $K$ sufficiently large

$$
E_{n}(\theta)=V\left(x_{\theta}, \theta\right)+(2 n+1) \sqrt{\frac{V^{\prime \prime}\left(x_{\theta}, \theta\right)}{2}}+O(1),
$$

where $x_{\theta}$ is the critical point of $V(\cdot, \theta)$ with $\mathfrak{R} x_{\theta} \in W$.

Remark 2. The error term in (5.3) is analytic in $\theta$ and the formula continues to hold after 2 formal differentiations. For our potential we get:

$$
\begin{gathered}
E_{n}(\theta+i \delta)=-2 K^{2}+\frac{K^{2}(\theta+i \delta)^{2}}{2\left(1+\alpha^{2}\right)}+O(K) \\
E_{n}^{\prime}(\theta+i \delta)=\frac{K^{2}(\theta+i \delta)}{1+\alpha^{2}}+O(K) \\
E_{n}^{\prime \prime}(\theta+i \delta)=\frac{K^{2}}{1+\alpha^{2}}+O(K)
\end{gathered}
$$

Note that the above implies $E_{n}(\theta)$ has a unique critical point $p_{n}$ with $\left|p_{n}\right|<$ const. $K^{-1}$. 
Fig. 2. $\mathscr{E}$ lies between the hatched regions $I_{n}$

Remark 3. If $E_{n}^{k}(\theta)$ is the $n^{\text {th }}$ eigenvalue of $H_{W}$ for the $k^{\text {th }}$ well, then

$$
E_{n}^{k}(\theta)=E_{n}(\theta-2 \pi k \alpha) \text {. }
$$

In particular, their critical values are the same. This follows from the fact that the wells are translates of each other by $2 \pi k$ and

$$
V(x-2 \pi k, \theta)=V(x, \theta-2 \pi k \alpha) .
$$

In order to obtain a priori bounds on $G_{A}$ we will need to know that $\Im E_{n}(\theta+i \delta) \neq 0$ for $\delta \neq 0$. Since

$$
E_{n}(\theta+i \delta)=E_{n}(\theta)+E_{n}^{\prime}(\theta) i \delta+O\left(K^{2} \delta^{2}\right)
$$

we need only avoid the energies near the critical values of $E_{n}$.

\section{Definition 3.}

$$
\mathscr{E} \stackrel{\text { def }}{=}[\inf \sigma(H), \inf \sigma(H)+200 K] \backslash \cup I_{n},
$$

where $I_{n}$ is an interval of width $2 K^{-2}$ centered at $E_{n}\left(p_{n}\right)$ - the critical value of $E_{n}(\theta)$. See Fig. 2. (Recall that since $\alpha$ is irrational $\sigma(H(\theta))$ is independent of $\theta$ a.e.)

Proposition 6. For all $E \in \mathscr{E}$,

$$
\left|E_{n}^{k}(\theta+i \delta)-E\right|>\text { const. } \delta .
$$

Proof. In view of Remark 3 we may assume that $k=0$. We have:

$$
E_{n}^{\prime}(\theta)=E_{n}^{\prime}\left(p_{n}\right)+E_{n}^{\prime \prime}(\xi)\left(\theta-p_{n}\right)=E_{n}^{\prime \prime}(\xi)\left(\theta-p_{n}\right),
$$

where $p_{n}$ is the critical point of $E_{n}$ and $\xi \in\left[\theta, p_{n}\right]$. It follows from (5.12) that

$$
\begin{aligned}
E_{n}(\theta)-E_{n}\left(p_{n}\right) & =\frac{1}{2} E_{n}^{\prime \prime}\left(\xi_{1}\right)\left(\theta-p_{n}\right)^{2} \\
& =\frac{1}{2} E_{n}^{\prime \prime}\left(\xi_{1}\right)\left[\frac{E_{n}^{\prime}(\theta)}{E_{n}^{\prime \prime}(\xi)}\right]^{2}=\left[\frac{1+\alpha^{2}}{2 K^{2}}+O\left(K^{-3}\right)\right]\left[E_{n}^{\prime}(\theta)\right]^{2} .
\end{aligned}
$$

If $\left|E_{n}^{\prime}(\theta)\right| \geqq\left(1+\alpha^{2}\right)^{-1 / 2}$, then (5.9) implies that

$$
\left|E_{n}(\vartheta)-E\right| \geqq\left|\Im E_{n}(\vartheta)\right| \geqq \text { const. } \delta,
$$

where $\vartheta$ denotes $\theta+i \delta$. If, on the other hand, $\left|E_{n}^{\prime}(\theta)\right| \leqq\left(1+\alpha^{2}\right)^{-1 / 2}$, then by (5.13) and (5.9),

$$
\left|E_{n}(\vartheta)-E_{n}\left(p_{n}\right)\right|<\frac{1}{2 K^{2}}+O\left(K^{-3}\right) .
$$

In particular,

$$
\operatorname{dist}\left[E_{n}(\vartheta), \mathscr{E}\right]>\frac{1}{3 K^{2}}>\text { const. } \delta
$$




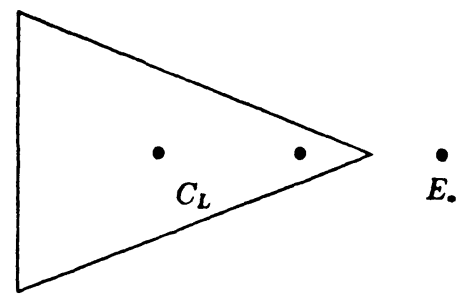

Fig. 3. $\sigma\left(H_{W}\right)$ and the contour $C_{L}$

Having established that $\sigma\left(H_{W}\right)$ stays away from $E$ for $\delta>0$ we prove below that $\left\|G_{W}\right\| \leqq 2\left[\operatorname{dist}\left(\sigma\left(H_{W}\right), E\right)\right]^{-1}$. This inequality holds since for $\delta$ small $H_{W}(\theta+i \delta)$ is near a self-adjoint operator.

Let $\mathfrak{R} H_{W} \stackrel{\text { def }}{=}-D^{2}+\mathfrak{R} V$ be an operator on $L^{2}(W)$. It is self-adjoint, has discrete spectrum, and its eigenvalues $\tilde{E}_{n}$ are simple. Moreover, they are very close to the $E_{n} \mathrm{~s}$ :

Lemma 6. Let $E_{n}$ denote the $n^{\text {th }}$ eigenvalue of $H_{W}$. Then for $\delta<K^{-3}$,

$$
\left|E_{n}-\tilde{E}_{n}\right| \leqq 3 K^{2} \delta
$$

Proof.

$$
\|\Im H\|=|\Im V|=\left|K^{2} \sinh \delta \sin (\alpha x+\theta)\right| \leqq 2 K^{2} \delta .
$$

In other words, $H_{W}$ is a small-norm perturbation of $\mathfrak{R} H_{W}$. Equation (5.17) follows from the standard arguments of perturbation theory. See [17].

Let us choose $\delta \leqq K^{-5}$. From Lemma 6 we get:

$$
\left|E_{n}-E_{m}\right|>K \quad \forall n \neq m ; \quad n, m \leqq 100 K .
$$

This allows us to partition $H_{W}$ into 3 parts. Let $E_{*}$ be the eigenvalue of $H_{W}$ nearest $E$. (If there are 2 , we pick the one with smaller real part.) Let

$$
P_{*} \stackrel{\text { def }}{=} \frac{-1}{2 \pi i} \int_{\left|z-E_{*}\right|=K / 4} \frac{d z}{H_{W}-z} \quad \text { and } \quad P_{L} \stackrel{\text { def }}{=} \frac{1}{2 \pi i} \int_{C_{L}} \frac{d z}{H_{W}-z}
$$

be the projections onto the subspaces corresponding to $E_{*}$ and to the eigenvalues lower than $E_{*}$, respectively. Define $P_{U}=1-P_{L}-P_{*}$. See Fig. 3. $P_{*}$ and $P_{L}$ commute with $H_{W}$, and $P_{*} P_{L}=0=P_{L} P_{*}$. It follows that $P_{U}$ is also a projection, commutes with $H_{W}$, and $P_{U} P_{*}=P_{*} P_{U}=P_{U} P_{L}=P_{L} P_{U}=0$.

Let $G_{L}(E) \stackrel{\text { def }}{=}\left(P_{L} H_{W}-E\right)^{-1}$ and $G_{U}(E) \stackrel{\text { def }}{=}\left(P_{U} H_{W}-E\right)^{-1}$. Then

\section{Lemma 7.}

$$
\left|G_{L}(E)+G_{U}(E)\right| \leqq \frac{4}{K}
$$

Proof. Lemma 6 implies that $E_{n}$ lies in the interior of $C_{L}$ iff $\tilde{E}_{n}$ does. Since $\mathfrak{R} H_{W}$ is self-adjoint

$$
\left\|P_{L} \frac{1}{\mathfrak{R} H_{W}-E}\right\| \leqq \frac{1}{\operatorname{dist}\left(E, \sigma\left(\Re H_{W} P_{L}\right)\right)} \leqq \frac{1}{K}
$$


Therefore,

$$
\left\|P_{L} \frac{1}{H_{W}-E}\right\|=\left\|P_{L} \frac{1}{\mathfrak{R} H_{W}-E} \cdot \frac{1}{1+\left(\mathfrak{R} H_{W}-E\right)^{-1} \mathfrak{J} H_{W}}\right\| \leqq \frac{2}{K}
$$

for $\delta$ sufficiently small. The estimate on $G_{U}(E)$ is the same.

Lemma 8. Let $\phi \in L^{2}(W)$ be the normalized eigenfunction of $H_{W}$ with eigenvalue $E_{*}$ closest to $E$ and $G_{W}(x, y, E)$ be the Green's function. Then, if $E \notin \sigma\left(H_{W}\right)$,

$$
G_{W}(x, y, E)=\frac{\phi_{*}(y) \phi(x)}{E_{*}-E}+O\left(\frac{1}{K}\right),
$$

where $\phi_{*}$ is the normalized eigenfunction of $H^{*}$ with eigenvalue $\bar{E}_{*}$.

Proof. Let $f, g \in L^{2}(W)$ be any test-functions with unit $L^{2}$ norm. Then

$$
\int d x d y \overline{f(x)} G_{W}(x, y, E) g(y)=\left\langle f, G_{W} g\right\rangle=\left\langle f, G_{W} P_{*} g\right\rangle+\left\langle f, G_{W}\left(P_{L}+P_{U}\right) g\right\rangle .
$$

The second term is bounded by:

$$
\left|\left\langle f, G_{W}\left(P_{L}+P_{U}\right) g\right\rangle\right| \leqq\|f\|_{2}\|g\|_{2}\left(\left\|G_{W} P_{L}\right\|+\left\|G_{W} P_{U}\right\|\right) \leqq \frac{4}{K}
$$

and becomes the error term in (5.24). To compute the first term we note that

Then

$$
P_{*} g=\left\langle\phi_{*}, g\right\rangle \phi \text {. }
$$

$$
\left\langle f, G_{W} P_{*} g\right\rangle=\left\langle\phi_{*}, g\right\rangle\left\langle f, G_{W} \phi\right\rangle=\frac{\left\langle\phi_{*}, g\right\rangle\langle f, \phi\rangle}{E_{*}-E} .
$$

Since $\phi, \phi_{*}$, and $G_{W}(x, y, E)$ are continuous, letting $f$ tend to $\delta_{x}, g$ to $\delta_{y}$ and combining (5.25), (5.26), and (5.28) we get (5.24).

In order for Proposition 8 to be useful we need to bound $\|\phi\|_{\infty}$.

Lemma 9. Let $\phi$ be as in Proposition 8. Then

$$
\|\phi\|_{\infty} \leqq 2 K^{4 / 5} \text {. }
$$

Proof. Let $x \in W$ and $w \in \partial W$. Since $\phi(w)=0$,

$$
\begin{aligned}
|\phi(x)| & \leqq \int_{w}^{x}\left|\phi^{\prime}(t)\right| d t \\
& \leqq|x-w|^{1 / 2}\left|\left\langle\phi^{\prime}, \phi^{\prime}\right\rangle\right|^{1 / 2} \\
& \leqq|W|^{1 / 2}\left|\left\langle\phi, \phi^{\prime \prime}\right\rangle\right|^{1 / 2}=|W|^{1 / 2}|\langle\phi,(V-E) \phi\rangle|^{1 / 2} \\
& \leqq|W|^{1 / 2} 2 K\|\phi\|_{2} \leqq 2 K^{4 / 5}
\end{aligned}
$$

since $|W|=K^{-2 / 5}$ and $\|\phi\|_{2}=1$.

We are now sufficiently armed to bound $G_{W}$. 
Proposition 7. For $E \in \mathscr{E}$,

$$
\left|G_{W}(x, y, E)\right|<\frac{K^{2}}{\delta}
$$

for all $x, y \in W$.

Proof. Combining Lemma 9, Lemma 8, and Proposition 6 we get:

$$
\left|G_{W}(x, y, E)\right| \leqq \text { const. } \frac{K^{8 / 5}}{\delta}<\text { const. } \frac{K^{2}}{\delta} \quad \forall x, y \in W .
$$

Outside the wells $E$ lies below the minimum of $\mathfrak{R} V$ and, therefore, the Green's function decays. The next lemma contains the precise statement.

Lemma 10. Let $\Lambda$ be any interval such that

$$
\min _{\Lambda} \mathfrak{R} V-\max \mathscr{E}>K^{6 / 5} / 2
$$

Then for all $x, y \in \Lambda$ with $\operatorname{dist}(\{x, y\} ; \partial \Lambda)>K^{-1 / 3}$,

$$
\begin{aligned}
G_{\Lambda}(x, y)= & -\frac{1}{2}(V-E)^{-1 / 4}(x)(V-E)^{-1 / 4}(y) \exp \left\{-\int_{x}^{y}|V-E|^{1 / 2}\right\} \\
& \cdot\left\{1+O\left(K^{-1 / 5}|\Lambda|\right)\right\} .
\end{aligned}
$$

Remark 4. If $\Lambda$ contains no wells condition (5.36) holds.

Proof. Let $p$ be a point in the middle of $\Lambda$. It is a standard result of WKB theory (see [15]) that the O.D.E.

has 2 solutions

$$
u^{\prime \prime}=(V-E) u
$$

$$
\phi_{+}(y)=(V-E)^{-1 / 4}(y) \exp \left\{\int_{p}^{y}(V-E)^{1 / 2}\right\}\left\{1+\varepsilon_{+}(y)\right\}
$$

and

$$
\phi_{-}(y)=(V-E)^{-1 / 4}(y) \exp \left\{\int_{p}^{y}-(V-E)^{1 / 2}\right\}\left\{1+\varepsilon_{-}(y)\right\}
$$

such that

$$
\left|\varepsilon_{ \pm}(y)\right|,\left|(V-E)^{-1 / 2}(y) \varepsilon_{ \pm}^{\prime}(y)\right| \leqq \text { const. } K^{-1 / 5}|\Lambda| .
$$

Since the Green's function can be expressed explicitly in terms of these functions (5.37) follows from a straightforward computation.

We have now established the decay and the a priori bounds. It remains to patch the results together. This will be done in Proposition 8 as soon as we prove the next lemma.

Lemma 11. Let $G$ be a Green's function in any box $\Lambda$ larger than $K^{-1 / 2}$, finite or infinite and $\left|G^{\bullet}(x, y)\right| \leqq$ const. $e^{-K|x-y|}$. Then, for $y_{0} \in \partial \Lambda$ and $\left|x-y_{0}\right|>1$,

$$
\left|G^{\bullet}\left(x, y_{0}\right)\right| \leqq \text { const. } K\left|x-y_{0}\right| e^{-K\left|x-y_{0}\right|}
$$




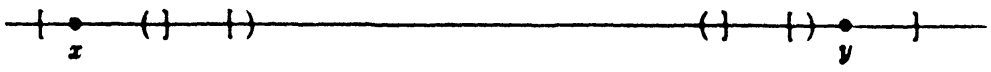

Fig. 4. $R_{j}$ s lie between square brackets and $W_{i}$ s between parentheses

Proof. Let $G_{0}(x, y, E)$ be the free Green's function, i.e., with $V \equiv 0$. Since $E=-2 K^{2}+o\left(K^{2}\right)$

$$
\left|G_{0}(x, y, E)\right|<\frac{e^{-K|x-y|}}{K} \text { and }\left|G_{0}^{\bullet}(x, y, E)\right|<e^{-K|x-y|} .
$$

Differentiating the identity

$$
G(x, y)=G_{0}(x, y)-G V G_{0}(x, y)
$$

with respect to $y$, we get:

$$
\begin{aligned}
G^{\bullet}(x, y) & =G_{0}^{\bullet}(x, y)-G V G_{0}^{\bullet}(x, y) \\
& =G_{0}^{\bullet}(x, y)-\int G(x, t) V(t) G_{0}^{\bullet}(t, y) d t .
\end{aligned}
$$

Therefore,

$$
\begin{aligned}
\left|G^{\bullet}(x, y)\right| & \leqq e^{-K|x-y|}+3 K^{2} \int|G(x, t)| e^{-K|t-y|} d t \\
& \leqq \text { const. } e^{-K|x-y|}+\text { const. } K e^{-K|x-y|}|x-y|
\end{aligned}
$$

In the following proposition we combine the local information provided by Proposition 7 and Lemma 10 to prove the decay of $G_{A}$. This will be done using block-resolvent expansion, [21], which can be thought of as a random walk expansion with steps the size of the blocks. (These sizes need not be equal.) For our blocks we take the wells $\left\{W_{i}\right\}$ and the "decay regions" $\left\{R_{j}\right\}$. The decay in $\left\{R_{j}\right\}$ will offset the size of the Green's function in the wells.

Proposition 8. Let $\Lambda$ be a long interval, $x, y \in \Lambda$ with $\operatorname{dist}(\{x, y\}, \partial \Lambda)>K^{-1 / 3}$, and $|x-y|>K^{-1 / 3}$. Then for $E \in \mathscr{E}$

$$
\left|G_{\Lambda}(x, y, E ; \theta+i \delta)\right|<\text { const. } e^{-K|x-y|} .
$$

Proof. Let the intervals $W_{i} \subset \Lambda$ be the wells and let $R_{i} \subset \Lambda$ be the "decay regions" satisfying the hypotheses of Lemma 10 . Let $\mathscr{C} \stackrel{\text { def }}{=}\left\{W_{i}, R_{j}\right\}$ be a open cover of $\Lambda$. (See Fig. 4.) They can be chosen so that

1. $\cup C_{i} \supset \Lambda$.

2. Every point of $\Lambda$ belongs to at most two members of $\mathscr{C}$. In particular, a boundary point of each member belongs to the interior of exactly one member.

3. If $a, b \in \cup \partial C_{i}$ then $|a-b|>K^{-1 / 3}$ unless $a=b$. We also ask that $\operatorname{dist}(\{x, y\}$, $\{a, b\})>K^{-1 / 3}$.

In addition we ask that $R_{j}$ s are as long as Lemma 10 allows, i.e., either $R_{j}$ intersects two wells or $\partial \Lambda$, or $\left|R_{j}\right|=$ const. $K^{1 / 5}$.

We are finally ready to start the expansion. For $x \in \Lambda$ let $C(x) \in \mathscr{C}$ be any element 
of $\mathscr{C}$ containing $x$. (If $x \in \cup \partial \mathscr{C}_{i}$, then $C(x)$ is unique.) By the resolvent identity (4.17)

$$
\begin{aligned}
G_{\Lambda}(x, y) & =G_{C(x)}(x, y)+\sum_{p_{1} \in \partial C(x)} G_{C(x)}^{\bullet}\left(x, p_{1}\right) G_{\Lambda}\left(p_{1}, y\right) \\
& =\sum_{p_{1}} G_{C(x)}^{\bullet}\left(x, p_{1}\right) G_{\Lambda}\left(p_{1}, y\right)
\end{aligned}
$$

because $y \notin C(x)$. Applying the resolvent identity to $G_{\Lambda}\left(p_{1}, y\right)$ and then to $G_{\Lambda}\left(p_{2}, y\right)$ and so on, we get

$$
G_{\Lambda}(x, y)=\sum_{p_{1}, \ldots, p_{n}} G_{C(x)}^{\bullet}\left(x, p_{1}\right) G_{C\left(p_{1}\right)}^{\bullet}\left(p_{1}, p_{2}\right) \cdots G_{\Lambda}\left(p_{n}, y\right)
$$

so long as $y \notin C\left(p_{k}\right)$ for $k<n$. Let us say $C\left(p_{n}\right) \ni y$. Then after one more step

$$
\begin{aligned}
& G_{\Lambda}(x, y) \\
& \quad=\sum_{p_{1}, \ldots, p_{n}} G_{C(x)}^{\bullet}\left(x, p_{1}\right) G_{C\left(p_{1}\right)}^{\bullet}\left(p_{1}, p_{2}\right) \cdots G_{C\left(p_{n-1}\right)}^{\bullet}\left(p_{n-1}, p_{n}\right) G_{C\left(p_{n}\right)}\left(p_{n}, y\right) \\
& \quad+\sum_{p_{1}, \ldots, p_{n}} G_{C(x)}^{\bullet}\left(x, p_{1}\right) G_{C\left(p_{1}\right)}^{\bullet}\left(p_{1}, p_{2}\right) \cdots G_{C\left(p_{n}\right)}^{\bullet}\left(p_{n}, p_{n+1}\right) G_{\Lambda}\left(p_{n+1}, y\right) .
\end{aligned}
$$

Continuing in this manner we express $G_{\Lambda}(x, y)$ as a sum of series of products with the sum having as many terms as visits to $C(y)$. Let us estimate a typical term of a series:

$$
G_{C_{(x)}}^{\bullet}\left(x, p_{1}\right) G_{C_{\left(p_{1}\right)}}^{\bullet}\left(p_{1}, p_{2}\right) \cdots G_{C_{\left(p_{n}\right)}}^{\bullet}\left(p_{n}, y\right) .
$$

If $C\left(p_{i}\right)$ is a well, we group $G_{C\left(p_{i}\right)}^{\bullet}$ with the next term $G_{C\left(p_{i+1}\right)}^{\bullet}$ which must be a decay region. (We shall assume for simplicity that $y$ lies in a decay region.) From Proposition 7, Lemma 10, and Lemma 11 it follows that

$$
\begin{aligned}
\left|G_{C\left(p_{i}\right)}^{\bullet}\left(p_{i}, p_{i+1}\right) G_{C\left(p_{i+1}\right)}^{\bullet}\left(p_{i+1}, p_{i+2}\right)\right| & \leqq \text { const. } \frac{K^{6}}{\delta} \exp \left\{-\int_{p_{i+1}}^{p_{i+2}}|V-E|^{1 / 2}\right\} \\
& \leqq \text { const. } \frac{K^{6}}{\delta} e^{-3 / 2 K\left|p_{i+2}-p_{i+1}\right|} \\
& \leqq e^{-4 / 3 K\left|p_{i+2}-p_{i+1}\right|}
\end{aligned}
$$

for $K$ large enough and $\delta$ not too small $\left(>e^{-K / 10}\right)$. Since $(5.57)$ is satisfied by each $G_{C\left(p_{j}\right)}^{\bullet}$ with $C\left(p_{j}\right)$ a decay region, and the total measure of the wells is a small fraction of $|x-y|$, we see that the expression in (5.56) is smaller than

$$
\text { const. } e^{-5 / 4 K|x-y|} \text {. }
$$

Moreover, condition 3 assures us that $\left|p_{i+1}-p_{i+2}\right|>K^{-1 / 3}$ and so (5.57) is less than

$$
\text { const. } e^{-K^{2 / 3}} \text {. }
$$

In other words, if we regard each factor in (5.56) corresponding to a well and its successor as a single factor, we can say that each factor is bounded by (5.59). Now,

$$
\left|G_{\Lambda}\left(x, y, E ; z_{0}\right)\right| \leqq 1 / \varepsilon \quad x, y \in \Lambda .
$$


Reinserting the series in (5.53) for each factor with $G_{\Lambda}$ until each term containing $G_{\Lambda}$ has at least const. $K^{-2 / 3} \log 1 / \varepsilon$ terms we see that indeed

$$
\left|G_{\Lambda}(x, y)\right| \leqq \text { const. } e^{-K|x-y|},
$$

where const. is independent of $\varepsilon$.

\section{Bound on Arg}

In this section $G$ will stand for $G_{\Lambda}$. We are going to prove that $\left|\underset{|z|=r}{\operatorname{Arg}} G_{\Lambda}\right|=$ $O(|x-y||\log \delta|)$. Recall that $\delta=\log (1 / r)$ and

$$
\underset{|z|=r}{\operatorname{Arg}} G(x, y)=\frac{1}{2 \pi i} \int \frac{\frac{d}{d z} G(x, y ; z)}{G(x, y ; z)} d z
$$

\section{Lemma 12.}

$$
\frac{d}{d z} G(x, y ; z)=\int_{-\infty}^{\infty} d t G(x, t ; z)\left\{\frac{d}{d z} V(t, z)\right\} G(t, y ; z) .
$$

Proof. Recall that $G=(H-E)^{-1}$. Therefore,

$$
\frac{d}{d z} G=G\left(\frac{d}{d z} H\right) G=G\left(\frac{d}{d z} V\right) G
$$

as desired.

It follows that

$$
\underset{|z|=r}{\operatorname{Arg}} G(x, y)=\frac{1}{2 \pi i} \int_{|z|=r} d z \int_{-\infty}^{\infty} d t \frac{G(x, t ; z) \frac{d}{d z} V(t, z) G(t, y ; z)}{G(x, y ; z)} .
$$

We separate the integration with respect to $t$ into 3 regions:

$$
\begin{aligned}
\text { Region I } & x<t<y, \\
\text { II } & t \leqq x, \\
\text { III } & t \geqq y .
\end{aligned}
$$

By resolvent identity (4.17), in region I $G(x, y ; z)=G_{\Lambda_{t}}^{\bullet}(x, t ; z) G(t, y ; z)$, where $\Lambda_{t}=\Lambda \cap(-\infty, t]$. Also, $G(x, t ; z)=G_{\Lambda_{t}}^{\bullet}(x, t ; z) G(t, t ; z)$. Therefore,

$$
\begin{gathered}
\int_{I} \frac{G(x, t ; z) \frac{d}{d z} V(t, z) G(t, y ; z)}{G(x, y ; z)} d t \\
\quad=\int_{x}^{y} G(t, t ; z) \frac{d}{d z} V(t, z) d t,
\end{gathered}
$$


which in absolute value does not exceed

$$
\begin{aligned}
& \sum_{W_{1} \subset[x, y]}\left\{\int_{W_{1}} d t\left|\frac{\phi_{*}^{i}(t) \phi^{i}(t)}{E_{*}^{i}-E} \frac{d}{d z} V(t, z)\right|+O(K)\right\} \\
& +\sum_{R_{1}} \in[x, y] \int_{R_{i}}\left|G(t, t ; z) \frac{d}{d z} V(t, z)\right| d t,
\end{aligned}
$$

where we used (5.24) in (6.10). By an argument similar to that of Proposition 8

$$
|G(t, t ; z)| \leqq\left|G_{R}(t, t ; z)\right|+\text { const. }
$$

Therefore, by Lemma 10 contribution from the last term is not greater than

$$
\begin{aligned}
& \sum_{i} \int_{R_{i}} 2 \sup _{z}|V(t, z)-E|^{-1 / 2}\left|\frac{d}{d z} V(t, z)\right| d t \\
& \quad \leqq \sum_{i} \text { const. } K^{-3 / 5} \cdot K^{2}\left|R_{i}\right| \leqq \text { const. } K^{2}|x-y| .
\end{aligned}
$$

Next we estimate (6.10). Let $W=W_{i}$ be the $i^{\text {th }}$ well in $[x, y]$. Since $\phi_{*}(t)=\bar{\phi}(t)$, $\|\phi\|_{2}=1$, and $|d / d z V| \leqq 2 K^{2}$,

$$
\begin{aligned}
& \left|\frac{1}{2 \pi i} \int d z \int d t \frac{\phi_{*}(t) \phi(t)}{E_{*}(\theta)-E} \frac{d}{d z} V(t, z)\right| \\
& \quad \leqq \frac{1}{2 \pi} \int \frac{r d \theta}{\left|E_{*}(\theta)-E\right|} \int d t|\phi(t)|^{2} 2 K^{2} \leqq \frac{K^{2}}{\pi} \int_{-\pi}^{\pi} \frac{d \theta}{\left|E_{*}(\theta)-E\right|} .
\end{aligned}
$$

From formulas (5.4) and (5.5) it follows that $\left|E^{\prime}\right|>1$ when $E(\theta) \notin \mathscr{E}$. Hence,

$$
\int_{-\pi}^{\pi} \frac{d \theta}{\left|E_{*}(\theta)-E\right|} \leqq \text { const. } \int_{-\pi}^{\pi} \frac{d \theta}{|\theta+i \delta|} \leqq \text { const. }|\log \delta| \text {. }
$$

Therefore,

$$
\text { const. } K^{2}|\log \delta|
$$

is the contribution to $(6.10)$ from a single well $W_{i}$. Summing over all the wells and adding the result to (6.13) we see that the contribution to $\operatorname{Arg} G$ from Region $I$ is less than

$$
\text { const. }|x-y|\left(K^{2}|\log \delta|+K^{2}\right) \text {. }
$$

Next we consider Region II: $t \leqq x$. We use the identity

$$
G(t, y ; z)=G_{\Lambda_{x}}^{\bullet}(t, x ; z) G(x, y ; z)
$$

to rewrite

$$
\int_{-\infty}^{x} d t \frac{G(x, t ; z) \frac{d}{d z} V(t, z) G(t, y ; z)}{G(x, y ; z)}
$$

as

$$
\int_{-\infty}^{x} d t G(x, t ; z) G_{\Lambda_{x}}^{\bullet}(t, x ; z) \frac{d}{d z} V(t, z)
$$


By the results of Sect. 5

$$
|G(x, t ; z)|,\left|G_{\Lambda_{x}}^{\bullet}(t, x ; z)\right| \leqq \text { const. } e^{-K|t-x|}
$$

so that $(6.21)$ converges and is less than const. $K^{2}$. Integration over the Region III is handled similarly.

Combining bounds (6.18) and (6.21), we conclude that

$$
|\operatorname{Arg} G(x, y)| \leqq \text { const. }|x-y|\left(K^{2}|\log \delta|\right)+O\left(K^{2}\right) .
$$

Therefore, the second term on the right-hand side in (4.25) is $O\left(K^{2} \delta|x-y||\log \delta|\right)$ and tends to 0 as $K \rightarrow \infty$. This implies that

$$
\frac{1}{2 \pi} \int_{0}^{2 \pi} d \theta \frac{\log \left|G_{\Lambda}\left(x, y, E ; e^{i \theta}\right)\right|}{|x-y|} \leqq-K+o(1)
$$

and $\gamma \geqq K+o(1)$.

\section{A Appendix}

Proposition 3. Let an interval $I \subset\left[\inf \sigma\left(H_{c}\right)\right.$, inf $\left.\sigma\left(H_{c}\right)+200 K\right]$ be longer than $e^{-K^{1 / 6}}$. Then

$$
\sigma\left(H_{c}\right) \cap I \neq \varnothing .
$$

Proof. Pick $E \in\left[\inf \sigma\left(H_{c}\right)\right.$, inf $\left.\sigma\left(H_{c}\right)+200 K\right]$. Choose a well $W_{k}$ so that the lowest eigenvalue $E_{0}^{k}(\theta)$ of the operator $H_{c}(\theta)$ on $L^{2}\left(2 \pi k-K^{-2 / 5}, 2 \pi k+K^{-2 / 5}\right)$ equals $E$ for some $\theta_{0}$. By standard perturbation theory [17] the harmonic oscillator approximation yields

$$
E_{0}^{k}(\theta)=V\left(x_{\theta}, \theta\right)+\sqrt{\frac{V^{\prime \prime}\left(x_{\theta}, \theta\right)}{2}}+O(1)
$$

and

$$
\left|\phi_{0}\left(x-x_{\theta}\right)\right| \leqq \text { const. } K^{1 / 4} e^{-K / 4\left|x-x_{\theta}\right|^{2}},
$$

where $"=\frac{d^{2}}{d x^{2}}$. We let $\theta=\theta_{0}$ and $\phi_{0}$ be the corresponding eigenfunction. Then,

$$
\begin{aligned}
\left|(H-E) \phi_{0}\right| & \leqq\left|\phi_{0}^{\prime \prime}\right|+\left|2 K^{2}+E\right|\left|\phi_{0}\right| \\
& \leqq\left(\left|x-x_{\theta}\right|^{2}+3 K^{2}\right) K^{1 / 4} e^{-K / 4\left|x-x_{\theta}\right|^{2}} \leqq \text { const. } e^{-K / 5\left|x-x_{\theta}\right|^{2}}
\end{aligned}
$$

Therefore,

$$
\left\|(H-E) \phi_{0}\right\|_{2} \leqq \text { const. } \int_{\left|x-x_{0}\right|>K^{-2 / 5}} e^{-K / 5\left|x-x_{\theta}\right|^{2}} d x \leqq \text { const. } e^{-K^{1 / 5} / 5},
$$

which implies that

$$
\operatorname{dist}\left(E, \sigma\left(H_{c}\right)\right) \leqq \frac{1}{\|(H-E)\|^{-1}} \leqq \text { const. } e^{-K^{1 / 5} / 5} .
$$


Acknowledgements. We would like to thank P. Deift, M. Herman, L. Pastur, and M. Rychlik for helpful discussions.

\section{References}

1. Albanese, C.: Quasiperiodic Schrödinger Operators with Pure Absolutely Continuous Spectrum. Preprint, CIMS

2. Aubry, S.: Solid State Sci. 8, 264 (1978)

3. Chulaevsky, V., Delyon, F.: Purely absolutely continuous spectrum for the almost Mathieu operators. J. Stat. Phys. 55, 1279-1284 (1989)

4. Chulaevsky, V., Sinai, Ya.: Anderson Localization for multi-frequency quasi-periodic potentials in one dimension. Commun. Math. Phys. 125, 91-112 (1989)

5. Craig, W., Simon, B.: Subharmonicity of the Liapunov index. Duke Math. J. 50, 551-560 (1983)

6. Delyon, F.: J. Phys. A 20, L21 (1987)

7. Dinaburg, E., Sinai, Ya.: The one-dimensional Schrödinger equation with a quasi-periodic potential. Funct. Anal. Appl. 9, 279-289 (1975)

8. Eliasson, L.: Floquet Solutions for the One-Dimensional Quasi-Periodic Schrödinger Equation. Preprint, University of Stockholm

9. Gordon, A.: Usp. Math. Nauk. 31, 257 (1976) [in Russian]

10. Fröhlich, J., Spencer, T., Wittwer, P.: Localization for a class of one-dimensional quasiperiodic potentials. Commun. Math. Phys. 132, 5-25 (1990)

11. Herman, M.: Une méthode pour minorer les exposants de Lyapunov et quelques exemples montrant le charactère local d'un théorème d'Arnold et de Moser sur le tore en dimension 2. Commun. Math. Helv. 58, 453-502 (1983)

12. Hiramoto, H., Kohmoto, M.: Phys. Rev. Lett. 62, 2714 (1989)

13. Kunz, H., Souillard, B.: Commun. Math. Phys. 78, 201-246 (1980)

14. Kingman, J. F. C.: Subadditive Processes, Lecture Notes in Mathematics, vol. 539. Berlin, Heidelberg, New York: Springer 1976

15. Olver, F. W. J.: Asymptotics and Special Functions. New York: Academic Press 1974

16. Pastur, L.: Spectral Properties of disordered systems in one-body approximation. Commun. Math. Phys. 75, 179 (1980)

17. Reed, M., Simon, B.: Methods of modern mathematical physics, vol. 1-4. New York: Academic Press 1982

18. Sarnak, P.: Spectral behavior of Quasi-periodic potentials. Commun. Math. Phys. 84, 377-401 (1982)

19. Sinai, Ya. G.: Anderson localization for one-dimensional difference Schrödinger operator with quasi-periodic potential. J. Stat. Phys. 46, 861-918 (1987)

20. Cycon, H. L. et al.: Schrödinger Operators. Berlin, Heidelberg, New York: Springer 1987

21. Spencer, T.: The Schrödinger equation with a random potential - A mathematical review. In: Critical Phenomena, Random Systems, Gauge Theories, Les Houches, XLIII. d Osterwalder K., Stora, R. (eds.)

22. Surace, S.: Trans. Am. Math. Soc. 320, 321 (1990)

Communicated by B. Simon 